\title{
ANÁLISIS DEL PERFIL Y COMPORTAMIENTO DE LOS HOGARES QUE COMPRAN EN CADENAS DE DISTRIBUCIÓN APLICANDO EL MODELO PROBABILÍSTICO LOGIT
}

ADRIÁN GUTIÉRREZ CABELLO UNIVERSIDAD NACIONAL DE SAN MARTÍN

VÍCTOR PÉREZ BARCIA UNIVERSIDAD NACIONAL DE SAN MARTÍN

ARGENTINA

"En esta civilización, donde las cosas importan cada vez más y las personas cada vez menos, los fines han sido secuestrados por los medios: las cosas te compran, el automóvil te maneja, la computadora te programa, la TV te ve." 
Recibido: 24/07/2013

Aceptado: 15/08/2013

\section{INTRODUCCIÓN}

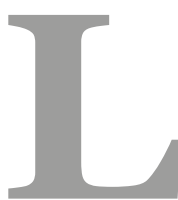

a Escuela de Economía y Negocios, (EEyN), de la Universidad Nacional de San Martín, (UNSAM), realiza estudios relacionados con los posibles impactos que, podrían producir la localización de nuevos establecimientos comerciales de las grandes cadenas, sobre los comercios y la población de cada uno de los centros urbanos en donde deseen instalarse estos emprendimientos, en el ámbito de la Provincia de Buenos Aires. Ello constituye un requisito imprescindible establecido por la Ley Provincial 12.573, para recibir la autorización de la instalación de estas cadenas.

Las investigaciones deben evaluar la viabilidad de la localización del Nuevo Emprendimiento Comercial, (NEC); si los resultados muestran que su instalación no causa distorsiones importantes en la estructura socioeconómica de la población, ni en los canales de comercialización establecidos, la EEyN-UNSAM eleva, a la Dirección Provincial de Comercio del Ministerio de Producción de la Provincia de Buenos Aires, un informe que sirve de base para la aprobación de la instalación del NEC en la localidad solicitada.

Los estudios que se realizan, si bien se evalúan con las modernas herramientas de análisis y estudios de campo, solamente constituyen proposiciones que deben ser verificadas a través de nuevas investigaciones. Ello permite contrastar los resultados obtenidos y la metodología utilizada con el impacto real que sucedió luego de la instalación del NEC.

El Modelo Probabilístico Logit, que aquí se expone, constituye uno de los principales instrumentos de análisis utilizados que permite fundamentar las conclusiones obtenidas.

Este trabajo es parte de una investigación realizada en la EEyN sobre el impacto que tuvieron la radicación de estos NEC en cinco localidades de la Provincia de Buenos Aires, siendo estas Roque Pérez, Bragado, Ituzaingó, Bolívar y Veinticinco de Mayo, En este caso se analizará el comportamiento que tuvieron los hogares. Se relevaron cuatrocientos hogares distribuidos entre las ciudades antes mencionadas, en la zona de influencia del $\mathrm{NEC}^{1}$.

\section{OBJETIVOS DEL ESTUDIO}

El objetivo del presente Proyecto es medir el impacto real producido en los hogares de las localidades seleccionadas como consecuencia de la instalación de los NECs (Nuevo Emprendimiento Comercial) como así también el perfil de los compradores actuales. En ese sentido, se realizan los estudios necesarios para ser contrastados empíricamente con las conclusiones presentadas en los EISEs (Estudios de Impacto Socio Económico) en oportunidad de su evaluación.

Ello permite considerar las propuestas efectuadas anteriormente, como así también analizar la metodología que fuera utilizada, a los efectos de convalidarla o bien sentar nuevas bases sobre las cuales implementar los futuros EISEs.

\footnotetext{
1 De acuerdo a la normativa vigente establece un radio de diez cuadras.
} 


\section{RESULTADOS OBTENIDOS}

Los principales elementos que surgen del Estudio de Pos Impacto Socio Económico (EPISE) permiten afirmar que son válidas las recomendaciones realizadas en los EISEs para las distintas localidades seleccionadas en el momento de su evaluación. Conviene destacar el hecho que, junto con las acciones que llevaron adelante localmente, la economía de la zona como la del país verificó un crecimiento sostenido que acompañó las medidas que aplicaron los comerciantes.

Asimismo, los resultados obtenidos en el tratamiento del conjunto de variables consideradas en los modelos e indicadores utilizados en la presente investigación, ponen de relieve también, la validez de la metodología suministrada en su oportunidad por las autoridades provinciales.

Los resultados verificados en los hogares, son similares a los obtenidos antes de la instalación del NEC. Ello puede ser explicado, en parte, debido al poco tiempo transcurrido entre ambas encuestas, dado que las estructuras sociales no reaccionan rápidamente, sino en períodos más largos.

Sin embargo, las variables que fundamentaron las conclusiones para la autorización de su instalación, luego de la apertura del NEC podrían haber sido susceptibles de cambio, como por ejemplo las distintas percepciones o actitudes que podrían haber adoptado los hogares respecto a la concurrencia a las distintas modalidades comerciales, las nuevas motivaciones de compra, la reconsideración en la evaluación de los beneficios y/o desventajas. Puede constatarse, que en general, las opiniones expresadas antes y después de la instalación en general no difieren significativamente.

El resto distribuye sus compras entre las distintas modalidades comerciales sin afectar significativamente a ninguna de ellas y en menor medida a los negocios tradicionales.

\section{Síntesis de los principales indicadores analizados.}

A continuación se analizan las variables más relevantes y los resultados obtenidos que permiten fundamentar estas conclusiones y recomendaciones.

En las decisiones de compra de los hogares intervienen el conjunto de modalidades comerciales. Por tal motivo las magnitudes que se indican a continuación, expresan la variedad de opciones de compra.

Se observa que el 69,8\% de los hogares realizan sus compras en supermercados (esto, como se ha dicho, no implica que solamente compren en él); seis de cada diez hogares compran en negocio de barrio; similar proporción concurre al NEC; siendo el negocio de centro comercial, el menos concurrido (51,2\%). Como puede observarse la mayor parte de los hogares no opta por hacer sus compras en una única modalidad comercial, sino que las realiza en todo tipo de comercio.

Los principales motivos de concurrencia de los hogares de acuerdo con las distintas modalidades comerciales son las siguientes: 
- El principal motivo de concurrencia al negocio de barrio, está dado, por la cercanía a los hogares; casi el 50\% compra por esta causa. Le siguen precio y trato personal. Este comportamiento se observa en los distintos estratos socioeconómicos de pertenencia de los hogares, de acuerdo con los resultados obtenidos a partir de la cuantificación en quintiles del ingreso de las familias.

- Los más importantes motivos de compra en el Centro Comercial son: la variedad de producto (29,9\%) seguido por la calidad de producto, (23,7\%) y el precio, (23,3\%). La variedad y calidad de producto constituyen los motivos más frecuentes en la decisión de compra independientemente del nivel de ingreso de los hogares.

- La decisión de compra en los supermercados está relacionada con los precios y la variedad de producto, entre ambos concentran alrededor del 77\%. Bastante más alejados le siguen en importancia los motivos cercanía y calidad de productos que acumulan un 17,2\%. En general, esta estructura de motivos de compra no se diferencia en forma marcada por la ubicación de las familias en los distintos quintiles de ingreso.

El principal motivo de concurrencia al NEC es:

- El principal motivo de compra son los precios que ofrece, (77\%), independientemente del nivel de ingreso de los hogares, siendo el resto de los motivos poco significativos.

Sin embargo conviene destacar cierta diferenciación existente entre la concurrencia al NEC y el ingreso del hogar:

- El 82,3\% de los hogares del primer quintil, compran en el NEC.

- En aquellos correspondientes al segundo y tercer quintil, la decisión de compra la asume entre un 60\% y 70\% de las familias. Asimismo, el motivo de compra de los hogares ubicados dentro de estos tramos de ingreso, además del precio son, el trato personal y la variedad de producto.

- En los últimos dos quintiles, que corresponden a las familias de ingresos más altos, la concurrencia es menor al 50\% de los hogares, el precio sigue siendo el motivo relevante de compra, siguiéndole en menor medida la calidad de producto, la falta de tiempo.

- Existe, en general, una estrecha relación entre la concurrencia al NEC y la distancia tanto medida en cuadras como en la percepción que tienen los hogares sobre su cercanía; cuánto más cerca se encuentra el hogar del NEC tanto mayor es su decisión de compra en él.

\section{CONSIDERACIONES}

Por último conviene señalar que la instalación del NEC implicó una merma en la facturación - que fue menor a la esperada - en los comercios existentes, pero que ese impacto no fue igual para todos los rubros: si bien seis de cada diez hogares son clientes del NEC sólo el 3,6\% compra exclu- 
sivamente allí, el resto distribuye sus compras entre las distintas modalidades comerciales.

Es bueno destacar que la estimación realizada sobre la caída de la cuota de mercado en los negocios tradicionales es del 5,6\%, mientras que en los autoservicios es de $7,5 \%$, valores estos que están por debajo a los previstos en el estudio ex - ante.

Los principales motivos del menor impacto son: las expectativas antes de la instalación no fueron satisfechas; la mejora de la situación económica general, mejora en los ingresos reales, precios competitivos, como así también la mejora en la calidad y de los servicios de los comerciantes ante el nuevo competidor, (oferta de nuevos productos y servicios, ampliación del horario de atención y reformas del local, entre otras actitudes).

\section{ANÁLISIS DEL PERFIL DE LOS CLIENTES DEL NEC A PARTIR DE UN MODELO PROBABILÍSTICO}

Se estableció un modelo para establecer cuál es la probabilidad de concurrencia al NEC a partir de las variables seleccionadas, abarcando aspectos económicos y sociales de los hogares, siendo estas: nivel de ingreso, influencia económica sobre el grupo familiar, nivel de educativo del jefe de hogar, si compra en un supermercado, cantidad de personas en el hogar y distancia en cuadras del hogar al nuevo establecimiento comercial.

Los principales resultados que pueden extraerse del modelo son que la principal motivación de compra en el NEC es la mejora de la economía del grupo familiar a partir de los menores precios que éste puede ofrecer. Este efecto se extiende sobre las demás modalidades comerciales que, a fin de poder ser competitivos, deben tener precios en un nivel cercano a los del NEC. Ello beneficia principalmente a los hogares de menores ingresos, que lo destinan una alta proporción a la compra de bienes de la canasta alimentaria.

Por otra parte es dable destacar que los autoservicios son los comercios que tienen una mayor competencia con la modalidad analizada. En consecuencia los negocios tradicionales no verían afectada de manera significativa su nivel de ventas, dado que uno de los principales motivos de compra de los hogares es la cercanía.

\section{ANÁLISIS DEL PERFIL Y COMPORTAMIENTO DE LOS HOGARES QUE COMPRAN O NO EN EL NEC APLICANDO EL MODELO PROBABILÍ́STICO LOGIT}

De acuerdo con el relevamiento que se llevó a cabo, aproximadamente seis de cada diez hogares ${ }^{2}$, concurren a los distintos NEC. En cambio cuando se los consultó sobre si comprarían ahí, el porcentaje de respuestas afirmativa fue del $86,6 \%$. Por lo tanto se observa una

${ }^{2}$ Estos hogares representan el 60\% de la población de las localidades seleccionadas. 
importante diferencia en la intención de compra ex ante y ex post a la instalación.

Por lo que se puede inferir es que existieron o existen motivaciones de compra que no fueron satisfechas por el NEC. Por lo tanto la intención de compra, anterior a la instalación esta sobreestimada con respecto a lo que realmente ocurrirá.

Para establecer el perfil de clientes que compran en el NEC, se realizó un Modelo Probabilístico Logit, para estimar cual es la probabilidad de concurrencia de acuerdo a las variables seleccionadas, estas abarcan tanto los aspectos económicos como sociales. La variable a explicar es si concurre o no al NEC, adoptando valor 1 en caso afirmativo y o en caso negativo.

\section{VARIABLE DEPENDIENTE DICOTÓMICA}

Supongamos que se quiere analizar la aceptación de cierto producto en un supermercado, en función del ingreso, sexo, nivel de educación, ubicación en la góndola, etc. Una persona puede o no aceptar el producto. Por lo tanto la variable endógena o dependiente puede adoptar solo dos valores:

$\cdot 1$ = aceptación

- $\mathrm{o}=$ rechazo

Como este ejemplo pueden encontrarse muchos más, tales como si una droga es efectiva o no para curar una enfermedad, la aceptación o rechazo de un préstamo, la participación o no de la mujer en el mercado laboral en función de ciertas variables explicativas.

Los métodos para estimar esta clase particular de modelos con variable dependiente dicotómica son:

- Modelo Lineal de Probabilidad (MLP).

- Modelo de Regresión Logística (Logit).

- Modelo de Regresión de Probabilidad (Probit).

De estos tres modelos el MLP es el más sencillo de utilizar, pero presenta algunos inconvenientes a la hora de su utilización, a saber:

- La no normalidad del término de error.

- Heterocedasticidad

- La probabilidad estimada puede estar fuera del intervalo de o-1.

Aunque se resuelvan estos problemas, el MLP no es un modelo atractivo dado que supone que las probabilidades condicionales aumenten linealmente con los valores de las variables explicativas. Es más probable que las probabilidades tiendan a reducirse a medida que los valores de las variables explicativas aumentan o se reduzcan indefinidamente. Por estos motivos en el presente trabajo utilizaremos el modelo logit. 


\section{MODELO DE REGRESIÓN LOGÍSTICA}

Un modelo de regresión con variable dependiente binomial, modelo logístico, será uno que permita estudiar si dicha variable discreta depende o no de otra u otras variables. Si una variable binomial de parámetro $\mathrm{p}$ es independiente de otra $\mathrm{X}$, se cumple:

$$
(p \mid X=x)=p
$$

Para cualquier valor $x$ de la variable $X$. Por lo tanto en este modelo y considerando una sola variable independiente $X$ se traduce en una función en la que p está dependiendo de $X$ y de los coeficientes cuya investigación permite tratar la relación de dependencia.

Si se cuenta con una única variable independiente X, el modelo de regresión logística sería:

$$
\ln (p / q) \mid X=x)=\alpha_{\mathrm{o}}+\alpha_{1} X
$$

Donde $\ln$ es logaritmo neperiano, $\alpha_{\mathrm{o}}$ y $\alpha_{1}$ son constantes y $X$ una variable que puede ser aleatoria o no, continua o discreta. El modelo se puede generalizar para $k$ variables independientes, para dar paso a un Modelo Logístico Múltiple.

$$
\ln (p / q) \mid=\alpha_{0}+\alpha_{1} X_{1}+\alpha_{2} X_{2}+\ldots+\alpha_{k} X_{k}
$$

Queda definido el modelo logístico como el logaritmo del $o d d s^{3}$ para el suceso que representa la variable aleatoria binomial puntual dependiente del modelo.

Odds $=$ Cociente de probabilidades $=$ Probabilidad de éxito $/$ Probabilidad de fracaso $=\mathrm{p} / \mathrm{q}$

Donde $\mathrm{q}=1-\mathrm{p}$

Otra forma de expresarlo es:

$\mathrm{OR}=$ probabilidad de un éxito

1- probabilidad de un éxito

Son varios los motivos por los cuales se plantea el modelo con el logaritmo del odds.

Uno de ellos es el campo de variación de $\ln (\mathrm{p} / \mathrm{q}$ ) ó z es todo el campo real, (de -4 a 4), mientras que, para p el campo es solo de o a 1 y para p/q es de $o$ a $\infty$.

$\mathrm{Al}$ modelo definido en función del logaritmo de odds no hay que ponerle restricciones a los coeficientes que dificultarían su estimación. Además en estos modelos los coeficientes son de fácil interpretación en términos de independencia o asociación entre las variables.

$$
\ln (p / q)=\alpha_{0}+\alpha_{1} X \Longleftrightarrow \ln \left(\frac{p}{1-p}\right)=e^{\alpha_{0}+\alpha_{1} X} \Rightarrow p=\frac{e^{\alpha_{0}+\alpha_{1} X}}{1+e^{\alpha_{0}+\alpha_{1} X}}=\frac{1}{1+e^{-\left(\alpha_{0}+\alpha_{1} X\right)}}
$$

${ }^{3}$ Odds Ratio (OR): Razón de Producto Cruzado o Cociente de Probabilidades, se utiliza para reportar la chance que tiene un individuo del grupo expuesto de tener el evento comparado a un individuo del grupo control. El OR nos da una medida de magnitud de la asociación entre la exposición y el evento. El odds ratio se utiliza en muchos casos como una buena aproximación al Riesgo Relativo, (RR) especialmente cuando se trata de estudios caso-control (en los cuales no se puede estimar riesgos) o a partir de modelos matemáticos multivariables como la regresión logística y como medida para resumir una revisión sistemática u otros estudios comparativos de tratamiento, diagnóstico, pronóstico. 
Estas expresiones permiten calcular la probabilidad del proceso binomial para los distintos valores de la variable X. La función de distribución logística queda representada de la siguiente forma:

$$
f(\mathrm{z})=\frac{1}{1+e^{-z}}
$$

El modelo de regresión logística modeliza la probabilidad de un proceso binomial como la función logística de una combinación lineal de la variable dependiente. La expresión para el modelo de regresión múltiple será:

$$
p=\frac{1}{1+e^{-\left(\alpha_{0}+\alpha_{1} X_{1}+\alpha_{2} X_{2}+\ldots+\alpha_{k} X_{k}\right)}}
$$

El modelo logit se puede interpretar que $\operatorname{los} \alpha_{1}$ a $\alpha_{\mathrm{k}}$, dependiendo de la cantidad de variables explicativas, miden el cambio en $p$ ocasionado por un cambio unitario en $\mathrm{X}$, por ejemplo si queremos calcular la probabilidad de tener un automóvil de acuerdo al ingreso, dice como el logaritmo de las probabilidades a favor de poseer un automóvil cambia a medida que el ingreso cambia en una unidad.

El intercepto $\alpha_{0}$ es el valor del logaritmo de las probabilidades a favor de tener un automóvil si el ingreso fuera cero. En el modelo MLP se supone que $p_{i}$ está linealmente asociado con $X_{i}$, en el modelo logit se supone que el logaritmo del cociente de probabilidades está relacionado con $X_{i}$. Lo que implica $p_{i}$ que no está relacionada linealmente con las variables explicativas.

\section{APLICACIÓN DEL MODELO LOGIT}

Como se mencionó anteriormente el modelo que presentaremos a continuación estima cual es la probabilidad de concurrencia a los nuevos establecimientos comerciales.

\section{VARIABLES}

Variable dependiente o endógena:

- Compra: Dicotómica. Tiene valor (1) si compra y (o) no compra.

Variables explicativas o exógenas:

- Quintil-(quintil): Categórica. Variable Proxy del ingreso. Los quintiles están definidos de acuerdo al ingreso per cápita del grupo familiar.

- Influencia económica-(infeco): Categórica. Indica cual fue la influencia sobre el grupo familiar la instalación del NEC, las categorías definidas son: positivamente, negativamente, en nada, no sabe.

- Educación-(educa): Categórica. Nivel educativo del jefe de hogar: sin instrucción o 
primario incompleto, primario completo, secundario incompleto, secundario completo, terciario o universitario completo y terciario o universitario completo.

- Supermercado-(super): Dicotómica. Si compra en un supermercado (1), No (o).

- Personas-(perso): Discreta. Cantidad de personas en el hogar.

- Distancia-(dista): Discreta. Distancia en cuadras del hogar al NEC.

Tras definir las variables, el modelo estaría planteado de la siguiente manera de acuerdo a la función de distribución logística acumulativa:

$$
f(\mathrm{z})=\frac{1}{1+e^{-z}}
$$

Reemplazando:

$$
\begin{aligned}
& f(\mathrm{z})=\frac{1}{1+e^{-\left(\alpha+\alpha_{1 i}{ }^{*} q u i n t i l{ }_{i}+\alpha_{2 i}{ }^{*} \text { infeco }{ }_{i}+\alpha_{3}{ }^{*} \text { super }+\alpha_{4 i}{ }^{*} \text { educa }{ }_{i}+\alpha_{5}{ }^{*}{ }^{*} \text { perso }+\alpha_{6}{ }^{*} \text { dista }\right)}}
\end{aligned}
$$

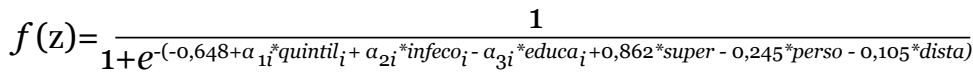

\section{RESUMEN DE LA REGRESIÓN LOGÍSTICA}

El siguiente cuadro indica las estimaciones de los parámetros, las variables explicativas son estadísticamente significativas, esto se puede observar en la columna -Sig. -, la única que no parece ser significativa es la categoría de negativa dentro de la variable influencia económica, pero es compensado con la alta significatividad de la categoría positiva, dentro de la misma variable. Los signos de las variables se corresponden con los esperados.

La columna $\operatorname{EXP(B),~da~la~derivada~del~cociente~de~probabilidades,~por~ejemplo~si~se~in-~}$ crementa en una cuadra la distancia al NEC, permaneciendo constante las demás variables, el “OR" pasará de 1 a 0,710 . 


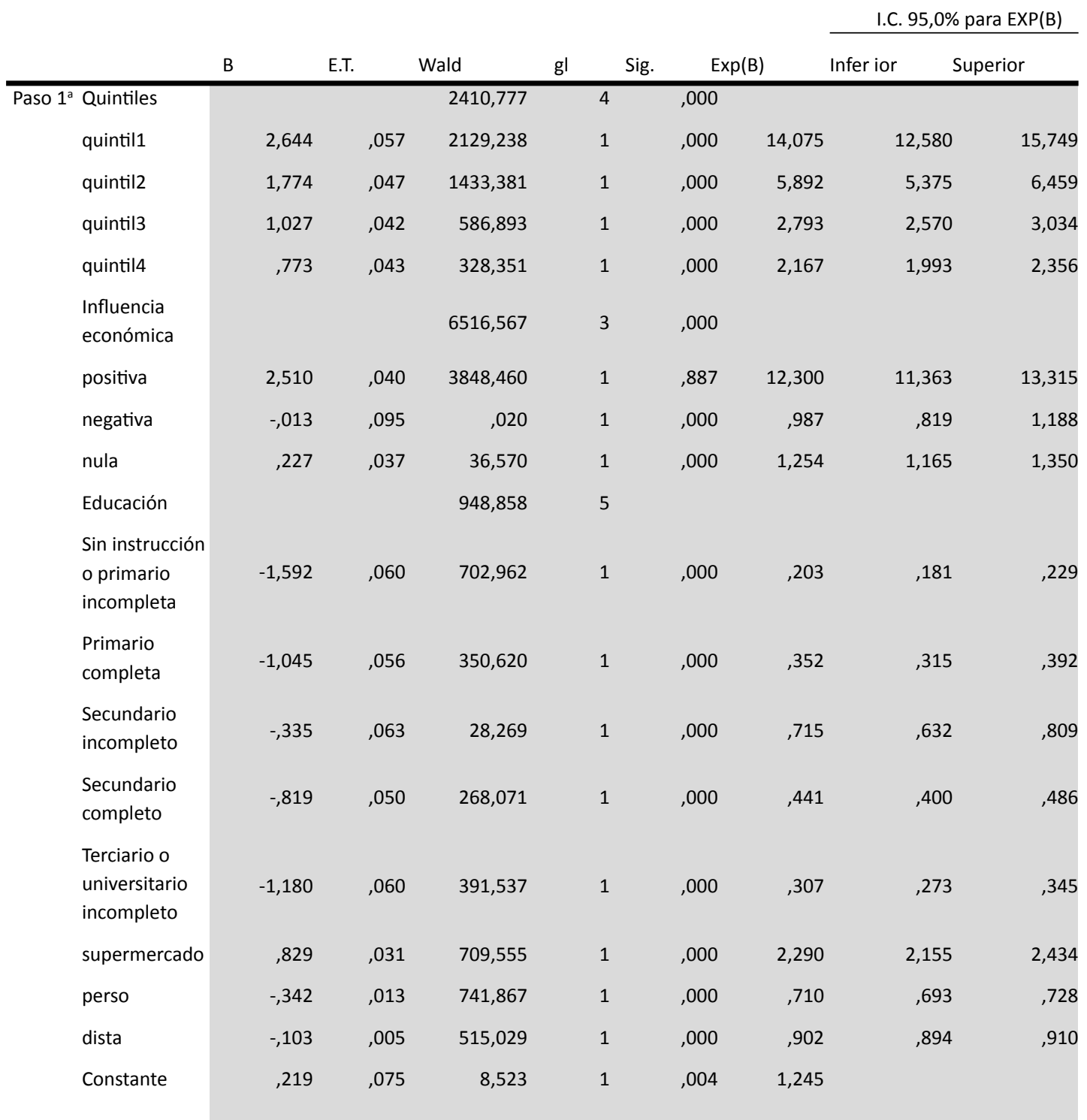

a. Variable(s) introducida(s) en el paso 1: Ningreso, P14, educa5, supermercado, P028, P011.

\section{ANÁLISIS DE LAS VARIABLES DEL MODELO.}

- Quintil: en caso de un hogar ubicado dentro del primer quintil su cociente de probabilidades pasa de 1 a 14,075 manteniendo constante las demás variables. El "B" disminuye a medida que aumenta el quintil de ingreso, por lo tanto disminuyen las preferencias de concurrencia al NEC si mayor es el ingreso del grupo familiar. 
- Influencia económica: Si el hogar considera que el NEC es positivo para la economía familiar también aumenta significativamente el OR, de esto se puede inferir que la compra en estos comercios depende principalmente de los precios, (variable que afecta el ingreso del grupo familiar).

- Supermercado: Haber tenido o tener el hábito de comprar en un supermercado también aumenta la probabilidad de concurrencia al NEC. Los hogares que compran en los negocios tradicionales tienen motivos de compra distintos a los que compran en los supermercados o en el NEC.

- Educación: Los hogares donde el jefe de familia tiene un nivel de instrucción medio tienen más probabilidades de concurrir que aquellos que tienen los niveles más bajos o más altos.

- Distancia: Si la distancia del hogar al NEC aumenta, disminuye la probabilidad de concurrencia.

- Personas: A medida que aumenta la cantidad de personas del hogar disminuye la probabilidad de concurrencia al NEC, los motivos que pueden llevar a esto que es que los hogares con más personas prefieren a los supermercados, dado que los hogares que concurren a estos tienen son más grandes que aquellos que concurren a los negocios tradicionales, (ya sea de barrio o centro comercial).

\section{CONTRASTE DE SIGNIFICACIÓN.}

En las regresiones logísticas para contrastar la significatividad global del modelo se utiliza el estadístico de la razón de verosimilitud, (RV). Este estadístico se distribuye como una Chi cuadrado con k-1 grados de libertad, siendo k el número de regresores incluyendo el término independiente.

$H_{\mathrm{o}}: \beta_{1}=\beta_{2}=\ldots=\beta_{k}=\mathrm{O}$

$H_{1}: N o H_{\mathrm{o}}$

$R V_{\mathrm{o}}: \chi_{k-1}^{2}$

El valor del estadístico Chi Cuadrado, con 15 grados de libertad y una significación del 1\% es 30,58, por lo tanto:

$\chi_{c}^{2}=\chi_{15 ; 0,01}^{2}=30,58$

$\chi_{o b s}^{2}=15.410,29 ; \chi_{c}^{2}<\chi_{o b s}^{2} \Rightarrow R H_{\mathrm{o}}$

Dado que el valor p, (significación), o (cero), el modelo en su conjunto es estadísticamente significativo.

\begin{tabular}{llrrrr}
\multicolumn{5}{c}{ Pruebas omnibus sobre los coeficientes del modelo } \\
& & Chi-cuadrado & gl & \multicolumn{3}{c}{ Sig } \\
\hline Paso 1 & Paso & 15410,296 & 15 &, 000 \\
& Bloque & 15410,296 & 15 &, 000 \\
& Modelo & 15410,296 & 15 &, 000
\end{tabular}




\subsection{5-BONDAD DE AJUSTE.}

A diferencia de la regresión lineal que se utilizan los coeficientes de determinación, en este caso aplicamos los Pseudos $\mathrm{R}^{2}$ que no pueden compararse con el $\mathrm{R}^{2}$ tradicional.

\begin{tabular}{lrrr}
\multicolumn{5}{c}{ Resumen de los modelos } \\
Paso & $\begin{array}{l}-2 \text { log de la } \\
\text { verosimilitud }\end{array}$ & $\begin{array}{l}\text { R cuadrado de } \\
\text { Cox y Snell }\end{array}$ & $\begin{array}{l}\text { R cuadrado de } \\
\text { Nag elkerke }\end{array}$ \\
\hline 1 & 36644,440 &, 329 &, 444
\end{tabular}

\footnotetext{
a. La est imación ha f inalizado en el número de iteración 5 porque las est imaciones de los parámetros han cambiado en menos de, 001 .
}

La capacidad de predicción del modelo la podemos observar en la tabla de clasificación. Para un valor de corte de $0,50^{4}$; el porcentaje pronosticado correcto es del $78,5 \%$, con un porcentaje mayor de predicción en aquellos que si concurren, (81,6\%). De esta manera tenemos el modelo que nos permite estimar cual es la probabilidad de comprar o no el NEC de acuerdo a las variables seleccionadas.

Si queremos estimar cual es la probabilidad que un hogar compre en el NEC de acuerdo a las siguientes características:

- quintil $_{\mathbf{1}}=$ Primer quintil de ingresos .

- infeco $_{1}=$ Positivamente.

- $\boldsymbol{E d u c a}_{2}=$ Primario completo .

- super = 1 (compra).

- perso = 4 (personas en el hogar).

- dista = 3 (distancia en cuadras del hogar al NEC).

$$
0,982=\frac{1}{1+e^{-\left(-0,219+2,644^{*} \text { quintil }_{1}+2,510^{*} *\right. \text { infeco }}{ }_{1}^{-1,045^{*} 0}+0,829^{*} 1_{\left.1-0,342^{*} 4-0,103^{*} 3\right)}}
$$

Por lo tanto el $p_{i}$ en este caso es del o,982 o expresado en porcentaje es del 98,2\%. El valor obtenido de la regresión original. Si queremos estimar el cociente de probabilidades, podemos calcular:

$$
O R=\frac{P_{i}}{1-P_{i}}=\frac{0,982}{1-0,982}=54,8
$$

La interpretación de este resultado sería que de acuerdo a los supuestos dados la posibilidad que un hogar compre contra uno que no es de 54,8 a 1 .

\footnotetext{
${ }^{4}$ El valor de corte por debajo de 0,5 asume supone la no concurrencia, por encima implica que si concurre.
} 
Probabilidad de concurrencia al NEC por quintil de ingreso

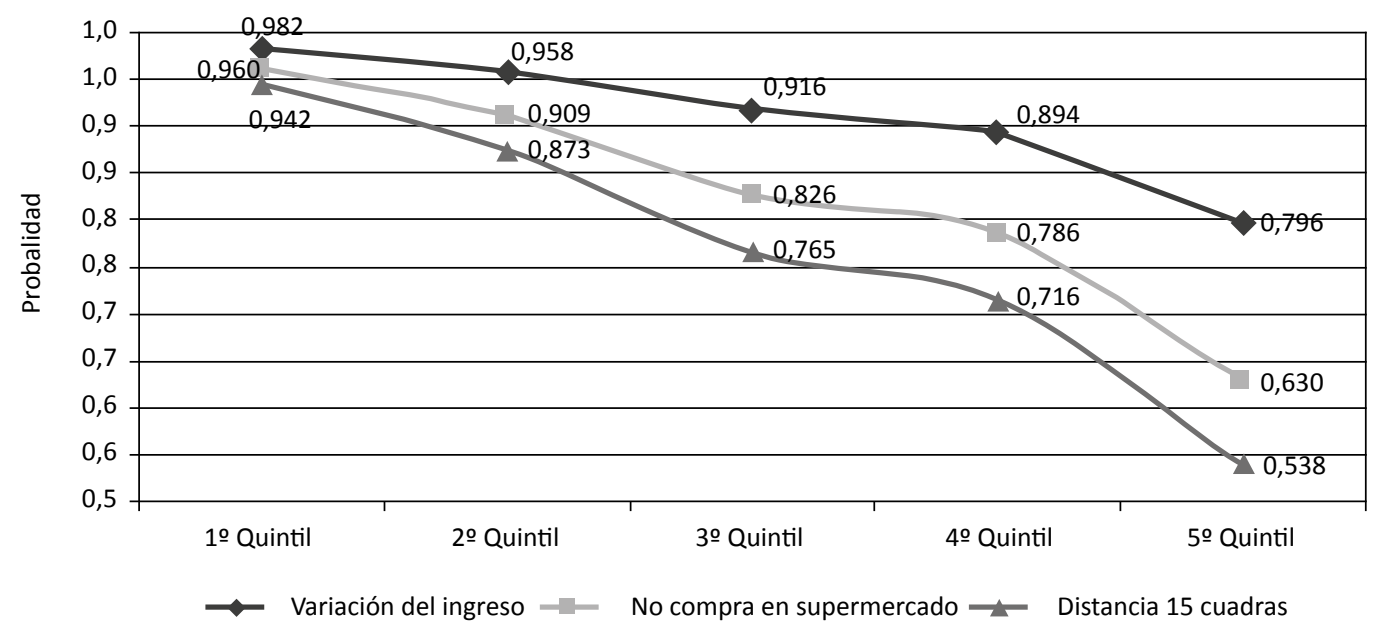

En un ejercicio de simulación podemos obtener las distintas probabilidades, variando alguno de los indicadores del ejemplo anterior. En el primer caso tenemos que el que influye positivamente sobre el hogar y la probabilidad estimada de acuerdo a cada quintil. En el segundo caso el hogar no compra en supermercados y en tercer lugar se cambió la distancia en cuadras, (15).

\section{CONCLUSIONES}

Se ve claramente la mayor probabilidad de concurrencia ocurre en los hogares de menores ingresos. Es interesante observar que los hogares que se encuentran a quince cuadras, pero consideran que es positivo el NEC para la economía familiar tienen una alta probabilidad de concurrencia, especialmente en el primero y segundo quintil de ingreso.

Del modelo podemos concluir que la principal motivación de compra en el NEC es la mejora de la economía del grupo familiar a partir de los menores precios que puede ofrecer éste. No menos importante es el efecto sobre las demás modalidades comerciales que afín de poder ser competitivos deben tener precios en un nivel cercano a los del NEC. Esto afecta principalmente a los hogares de menores ingresos donde lo destinan en una alta proporción a la compra de bienes de la canasta alimentaria. 
Además los autoservicios serían los comercios que tienen una mayor competencia con la modalidad analizada, por lo tanto los negocios tradicionales no verían afectada de manera significativa su nivel de ventas por esta modalidad comercial, donde uno de los principales motivo de compra es la cercanía.

Por lo tanto a priori la principal competencia se dará con los supermercados establecidos que tienen un formato similar al del NEC, en cambio la almacén tradicional no se vería afectada significativamente, a partir de un menor nivel de ventas.

\section{REFERENCIAS BIBLIOGRÁFICAS}

Cabrer Borrás, B. Sancho Pérez, A. Serrano Domingo, G. (2001) Microeconometría y decisión. Madrid: Ediciones Pirámide.

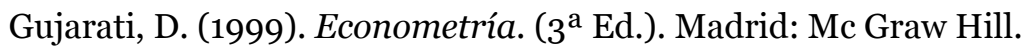

Pérez, C. (2003). Técnicas Estadísticas com SPSS. Madrid. Prentice Hall.

Provincia de Buenos Aires, Ministerio de Producción (2003). Ley Provincial 12.573 y sus modificaciones.

Provincia de Buenos Aires, Ministerio de Producción (2002) Instructivo Metodológico para la Medición de Impacto Socio Económico producido por la Instalación de Cadenas de Distribución en Localidades de la Provincia de Buenos Aires.

Uriel, G. \& Aldás, J. (2005) Análisis Multivariado Aplicado, Madrid: Thomson.

\section{CURRICULUM VITAE}

\section{Lic. Adrián Gutiérrez Cabello}

Curso de Alta Formación en "Gestión e Innovación en PyMEs. Universidad de Bologna-Italia. Lic. en Economía. Docente Adjunto concursado de Investigación de Mercado, y Seminario sobre economía regional. Investigador Categorizado Nivel III. Director de proyectos acreditados. Coordinador del Centro de Economía Regional de la EEyN-UNSAM. acabello@unsam.edu.ar 


\section{Lic. Víctor Pérez Barcia}

Especialista en programación económica Centre d' Etudes des programmes EconomiquesCEPE París. Licenciando en economía. Docente titular concursado de Administración Financiera y Mercado de Capitales (UNSAM). Investigador Principal del Centro de Economía Regional de la EEyN-UNSAM. Docente de Posgrado y jurado de concursos docentes. Director de proyectos acreditados.

Victor.PerezBarcia@unsam.edu.ar 